\title{
Galloping of wind-excited tower under external excitation and parametric damping
}

\author{
L. Mokni, I. Kirrou, M. Belhaq \\ Laboratory of Mechanics, University Hassan II-Casablanca, Morocco
}

\begin{abstract}
This paper investigates the influence of combined fast external excitation and fast parametric damping on the amplitude and the onset of galloping of a tower submitted to steady and unsteady wind flow. A lumped single degree of freedom model is considered and the cases where the turbulent wind activates either external excitation, parametric one or both are studied. The methods of direct partition of motion and the multiple scales are used to drive a slow dynamic near primary resonance. The influence of the combined excitations on the galloping is examined showing the beneficial effect on the amplitude and the onset of galloping when the excitations are introduced together.
\end{abstract}

\section{Introduction}

The control of large amplitude oscillations in tall flexible building constitutes an important issue in designing stable tall structures. Considerable efforts have been made to reduce the amplitude of oscillations induced by steady and unsteady wind. In general, taller flexible towers are aerodynamically sensitive to the natural wind environment and their failure may be expected if the nonlinear dynamic effects on the structure are neglected. It is well known that above a certain threshold of the wind speed, tall buildings develop galloping [1-5] causing the structure to oscillate with large amplitudes. In this context, considerable efforts have been done to quench such wind-induced oscillations including, for instance, mass tuned dampers, tuned liquid dampers or external excitation. A review of some control methods and their full-scale implementation to civil infrastructure applications is given in [6].

The effect of unsteady wind on the galloping onset of towers has been considered by several authors. In [4] a single degree of freedom (sdof) model has been considered and the multiple scales method (MSM) [7] has been used to analyze the influence of the unsteady wind on the critical wind speed above which galloping occurs. It was shown that the unsteady wind decreases significantly the galloping onset only near the primary resonance. In $[8,9]$, the effect of parametric, external and self-induced excitation on periodic galloping of a single tower and of two towers linked by a nonlinear viscous device was examined.

Recently, the effect of fast harmonic excitation (FHE) on periodic and quasiperiodic galloping onset of a tower exposed to steady and unsteady wind was studied near primary resonance [10]. The introduction of a FHE as a control strategy was motivated by an experimental work made for vibrating testing purpose of a full size tower [11]. The mechanical vibration exciter system used in such an experiment is placed on the top of the structure and debits a harmonic excitation to the structure. More recently, the influence of internal parametric damping (IPD) on periodic galloping onset of a tower under steady and unsteady wind was examined [12]. It was concluded that when the unsteady wind is present, IPD decreases the amplitude of galloping in all cases of loading, but has no influence on the galloping onset. Notice that the IPD can be introduced via a damper device in the interfloors damping as reported in [13]. Its use as a control strategy was motivated by its simple implementation and beneficial effect in reducing vibration in many engineering applications.

In this paper, we extend the results given in $[10,12]$ by focusing on the combined effect of external FHE and fast IPD on periodic galloping. The main purpose is to examine how FHE and IPD can influence the galloping of the wind-excited tower when both expiations are introduced simultaneously.

The paper is organized as follows: In Section 2, the equation of motion including the effect of both external excitation and parametric damping is given. The method of DPM [14,15] is performed and the MSM is applied in section 3 to derive the modulation equations of the slow dynamic near the primary resonance. In Section 4, we analyze the additional effect of FHE and IPD on the periodic galloping in the cases where the unsteady wind activates different excitations. Section 5 concludes the work.

\section{Equations of motion and slow dynamic}

A single mode approach of the tower motion is considered and a sdof lumped mass system is introduced $[4,8]$. It is assumed that the tower is subjected to steady and unsteady wind flow and to a combined effect of FHE and IPD. In this case, the dimensionless sdof equation of motion can be written in the form [8]

$$
\begin{array}{r}
\ddot{x}+x+\left[c_{a}(1-\bar{U})-b_{1} u(t)\right] \dot{x}+Y v^{2} \cos (v t) \dot{x}+b_{2} \dot{x}^{2} \\
+\left[\frac{b_{31}}{\bar{U}}+\frac{b_{32}}{\bar{U}^{2}} u(t)\right] \dot{x}^{3}=\eta_{1} \bar{U} u(t)+\eta_{2} \bar{U}^{2}+Y \cos (v t)
\end{array}
$$

where the dot denotes differentiation with respect to the non-dimensional time $t$. Equation (1) contains, in addition to the elastic, viscous and inertial linear terms, quadratic 
and cubic components in the velocity generated by the aerodynamic forces. The steady component of the wind velocity is represented by $\bar{U}$ and the turbulent wind flow is approximated by a periodic force, $u(t)$, which is assumed to include the two first harmonics, $u(t)=u_{1} \sin \Omega t+u_{2} \sin 2 \Omega t$, where $u_{1}, u_{2}$ and $\Omega$ are, respectively, the amplitudes and the fundamental frequency of the response. We shall analyze the case of external excitation, $u(t)=u_{1} \sin \Omega t$, parametric one, $u(t)=u_{2} \sin 2 \Omega t$, and the case where external and parametric excitations are present simultaneously. The coefficients of Eq. (1) are given in Appendix I and $Y, v$ are, respectively, the dimensionless amplitude and the frequency of the FHE and IPD. In order to simplify the analysis, one considers the particular case where the FHE and the IPD have the same amplitude and the same frequency.

Equation (1) includes a slow dynamic due to the steady and unsteady wind and a fast dynamic induced by the FHE and the IPD. To separate these dynamics, we perform the method of DPM on Eq. (1) by defining a fast time $T_{0}=v t$ and a slow time $T_{1}=t$, and splitting up $x(t)$ into a slow part $z\left(T_{1}\right)$ and a fast part $\phi\left(T_{0}, T_{1}\right)$ as

$$
x(t)=z\left(T_{1}\right)+\mu \phi\left(T_{0}, T_{1}\right)
$$

where $z$ describes the slow main motions at time-scale of oscillations, $\mu \phi$ stands for an overlay of the fast motions and $\mu$ indicates that $\mu \phi$ is small compared to $z$. Since $v$ is considered as a large parameter, we choose $\mu \equiv v^{-1}$ for convenience. The fast part $\mu \phi$ and its derivatives are assumed to be $2 \pi$-periodic functions of fast time $T_{0}$ with zero mean value with respect to this time, so that $\langle x(t)\rangle=$ $z\left(T_{1}\right)$ where $<>\equiv \frac{1}{2 \pi} \int_{0}^{2 \pi}() d T_{0}$ defines time-averaging operator over one period of the fast excitation with the slow time $T_{1}$ fixed. Averaging procedure gives the following equation governing the slow dynamic of motion

$$
\begin{array}{r}
\ddot{z}+z+\left[c_{a}(1-\bar{U})-b_{1} u(t)-H_{0}+\left(\frac{b_{31}}{\bar{U}}\right.\right. \\
\left.\left.+\frac{b_{32}}{\bar{U}^{2}} u(t)\right) H_{1}\right] \dot{z}+\left[B-B_{0}\left(\frac{b_{31}}{\bar{U}}+\frac{b_{32}}{\bar{U}^{2}} u(t)\right)\right] \dot{z}^{2}+\left[\frac{b_{31}}{\bar{U}}\right. \\
\left.+\frac{b_{32}}{\bar{U}^{2}} u(t)\right] H_{2} \dot{z}^{3}=\eta_{1} \bar{U} u(t)+\eta_{2} \bar{U}^{2}+G
\end{array}
$$

where $H_{0}=4 b_{2} Y^{2}, H_{1}=6\left(\frac{Y}{v}\right)^{2}, H_{2}=1+6 Y^{2} v^{2}, B=$ $b_{2}\left(1+2 Y^{2} v^{2}\right), B_{0}=12 Y^{2}$ and $G=-2 b_{2}\left(\frac{Y}{\gamma}\right)^{2}$. Note that the case without FHE has been studiend in [10], the case without IPD was considered in [12], while the case without FHE and IPD $(Y=0)$ was talked in [8].

\section{The multiple scales analysis}

To obtain the modulation equations of the slow dynamic (3) near primary resonance, the MSM is performed by introducing a bookkeeping parameter $\varepsilon$, scaling as $z=\varepsilon^{\frac{1}{2}} z$, $b_{1}=\varepsilon b_{1}, H_{0}=\varepsilon H_{0}, H_{1}=\varepsilon H_{1}, B=\varepsilon^{\frac{1}{2}} B, B_{0}=\varepsilon^{\frac{1}{2}} B_{0}$, $\eta_{1}=\varepsilon^{\frac{3}{2}} \eta_{1}, \eta_{2}=\varepsilon^{\frac{3}{2}} \eta_{2}$ and assuming that $\bar{U}=1+\varepsilon V$ [8]. With the resonance condition $\Omega=1+\varepsilon \sigma$ where $V$ stands for the mean wind velocity and $\sigma$ is a detuning parameter, a two-scale expansion of the solution is sought in the form

$$
z(t)=z_{0}\left(t_{0}, t_{1}\right)+\varepsilon z_{1}\left(t_{0}, t_{1}\right)+O\left(\varepsilon^{2}\right)
$$

where $t_{i}=\varepsilon^{i} t(i=0,1)$. In terms of the variables $t_{i}$, the time derivatives become $\frac{d}{d t}=d_{0}+\varepsilon d_{1}+O\left(\varepsilon^{2}\right)$ and $\frac{d^{2}}{d t^{2}}=$ $d_{0}^{2}+2 \varepsilon d_{0} d_{1}+O\left(\varepsilon^{2}\right)$, where $d_{i}^{j}=\frac{\partial^{j}}{\partial^{j} t_{i}}$. Substituting Eq. (4) into Eq. (3), equating coefficients of the same power of $\epsilon$, we obtain the two first orders of approximation

$$
\begin{gathered}
d_{0}^{2} z_{0}+z_{0}=G \\
d_{0}^{2} z_{1}+z_{1}=-2 d_{0} d_{1} z_{0}+\left(c_{a} V+b_{1} u\left(t_{0}\right)+H_{0}\right. \\
\left.-H_{1}\left(b_{31}+b_{32} u\left(t_{0}\right)\right)\right)\left(d_{0} z_{0}\right)-\left(B-B_{0}\left(b_{31}+b_{32} u\left(t_{0}\right)\right)\right)\left(d_{0} z_{0}\right)^{2} \\
\left.-H_{2}\left(b_{31}+b_{32} u\left(t_{0}\right)\right)\left(d_{0} z_{0}\right)^{3}+\eta_{1} u\left(t_{0}\right)+\eta_{2} 6\right)
\end{gathered}
$$

A solution of Eq. (5) is given by

$$
z_{0}=A\left(t_{1}\right) \exp \left(i t_{0}\right)+\bar{A}\left(t_{1}\right) \exp \left(-i t_{0}\right)+G
$$

where $i$ is the imaginary unit and $A$ is an unknown complex amplitude. Equation (6) can be solved for the complex amplitude $A$ by introducing its polar form as $A=\frac{1}{2} a e^{i \phi}$. Substituting the expression of $A$ into Eq. (6) and eliminating the secular terms, the modulation equations of the amplitude $a$ and the phase $\phi$ can be extracted as

$$
\left\{\begin{array}{l}
\dot{a}=\left[S_{1}-S_{3} \sin (2 \phi)\right] a-S_{5} \cos (\phi) a^{2} \\
+\left[-S_{2}+2 S_{4} \sin (2 \phi)\right] a^{3}-\beta \cos (\phi) \\
a \dot{\phi}=\left[\sigma-S_{3} \cos (2 \phi)\right] a+3 S_{5} \sin (\phi) a^{2} \\
+\left[S_{4} \cos (2 \phi)\right] a^{3}+\beta \sin (\phi)
\end{array}\right.
$$

where $S_{1}=\frac{1}{2}\left(c_{a} V+H_{0}-H_{1} b_{31}\right), S_{2}=\frac{3}{8} b_{31} H_{2}, S_{3}=$ $\frac{1}{4}\left(b_{1}-H_{1} b_{32}\right) u_{2}, S_{4}=\frac{1}{8} b_{32} H_{2} u_{2}, S_{5}=\frac{1}{8} b_{32} B_{0} u_{1}$ and $\beta=$ $\frac{\eta_{1} u_{1}}{2}$. It can be seen that the FHE and the IPD influence the dynamic of the tower via the coefficients $H_{i}(i=0,1,2)$ and $B_{0}$.

\section{Applications and results}

In this section, we analyze the effect of the amplitude $Y$ of the excitations on the vibration of the tower for different types of turbulent wind flow. The parameter values used in the present study are taken from [8].

\subsection{Case of non-turbulent wind}

Fixed points of Eq. (8), representing periodic oscillations of the system, are giving by setting $\dot{a}=\dot{\phi}=0$. In the absence of turbulence $\left(u_{1}=u_{2}=0\right)$, only the first equation of system (8) is used. Besides the trivial solution, $a=0$, the amplitude of the self-excitation is also obtained such as

$$
\left\{\begin{array}{l}
a=0 \\
a=\sqrt{\frac{4\left(c_{a} V+H_{0}-H_{1} b_{31}\right)}{3 b_{31} H_{2}}}
\end{array}\right.
$$

Figure 1 shows the galloping amplitude $a$ versus the wind velocity $V$ in the absence of the unsteady wind $\left(u_{1}=\right.$ $0, u_{2}=0$ ), as given by Eq. (9), for $\sigma=0$ and for different values of the amplitude $Y$ of the FHE and the IPD. The trivial solution exists everywhere and changes its stability at the bifurcation point. It can be seen from this figure that increasing the amplitude $Y$, the amplitude of the tower response decreases significantly and the location of the galloping onset shifts toward higher values of the steady wind velocity. 


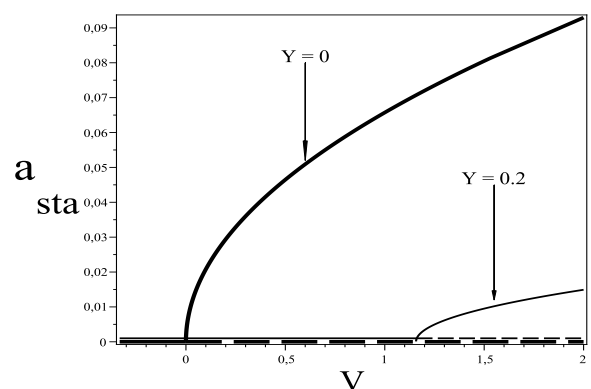

Fig. 1. Equilibrium branches in the absence of turbulent wind, for $v=8$. Solid line: stable, dashed line: unstable.

\subsection{Case where turbulent wind activates external excitation}

In this case $\left(u_{1} \neq 0, u_{2}=0\right)$, the modulation equations (8) lead to the following response equation

$$
\frac{\left(S_{1} a-S_{2} a^{3}\right)^{2}}{\left(\beta+S_{5} a^{2}\right)^{2}}+\frac{(-\sigma a)^{2}}{\left(\beta+3 S_{5} a^{2}\right)^{2}}=1
$$

In Figs. 2, 3, we show the effect of the amplitude $Y$ on the frequency response versus $V$ and $\sigma$, respectively, for different values of $Y$.

Figure 2 indicates that as the amplitude $Y$ increases, the amplitude of tower oscillation decreases and the frequency response shifts right. In Fig. 3 is shown the decreasing in the amplitude when $Y$ is increased. One can observe from this figure that for $Y=0$, three solutions exist within the interval $(\sigma \in[-0.0001,0.0001])$. As $Y$ is increased, only one solution exists. The solid lines correspond to the stable branches, while the dots correspond to the unstable ones. Validation of the analytical approximation is made using numerical simulation (circles).

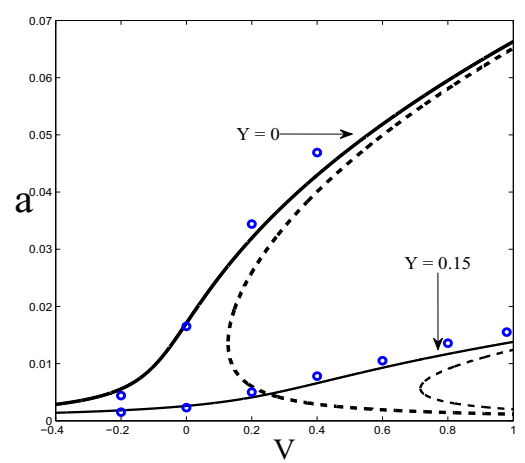

Fig. 2. Amplitude versus velocity of the wind when $\sigma=0, u_{2}=$ $0, u_{1}=0.1$ and $v=10$. Solid line: stable; dashed: unstable.

\subsection{Case where turbulent wind activates parametric excitation}

In this case $\left(u_{1}=0, u_{2} \neq 0\right)$, the corresponding amplitude response equation is written as

$$
\frac{\left(-S_{1} a+S_{2} a^{3}\right)^{2}}{\left(-S_{3} a+2 S_{4} a^{3}\right)^{2}}+\frac{(-\sigma a)^{2}}{\left(-S_{3} a+S_{4} a^{3}\right)^{2}}=1
$$

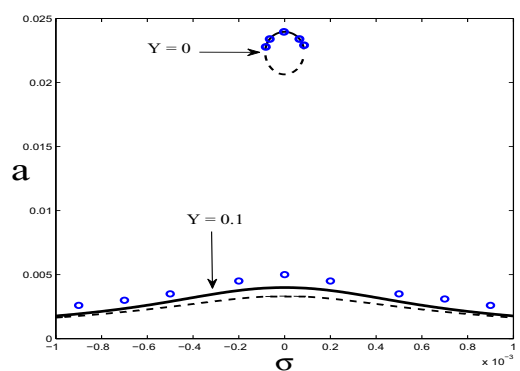

Fig. 3. Amplitude versus $\sigma$ when $V=0.117, u_{1}=0.033, u_{2}=$ 0 and $v=10$. Solid line: stable, dashed line: unstable, circles: numerical simulation.

Figure 4 shows the amplitude versus the velocity $V$, as given by (11), in the absence of external excitation $\left(u_{1}=\right.$ $\left.0, u_{2} \neq 0\right)$. The solid lines correspond to the stable branches, dots correspond to the unstable ones. The plots show that as $Y$ increases the amplitude of the response decreases significantly and a shift to the right in the frequency response is obtained. Figure 5 shows the decrease of the galloping amplitude versus $\sigma$ as $Y$ is increased.

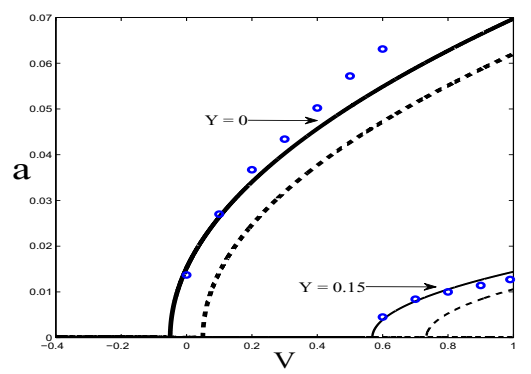

Fig. 4. Amplitude versus $V$ when $\sigma=0, u_{1}=0, u_{2}=0.1$ and $v=8$. Solid line: stable, dashed line: unstable, circles: numerical simulation.

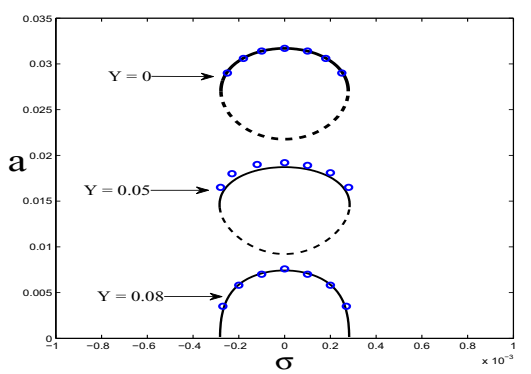

Fig. 5. Effect of $Y$ on galloping; $u_{2}=0.1, V=0.167, v=8$. Solid line: stable, dashed line: unstable, circles: numerical simulation.

\subsection{Case where turbulent wind activates external and parametric excitations}

In the case where the external and parametric excitations are both present $\left(u_{1} \neq 0, u_{2} \neq 0\right)$, the amplitude frequency response is shows in Fig. 6. The solid lines correspond to 
the stable branches, dots correspond to the unstable ones and circles are obtained by numerical integration. The plots indicate the effect the amplitude $Y$ on the frequency response indicating that increasing $Y$ eliminates the bistability in the amplitude response, thereby the coexistence of two different amplitudes of oscillation.

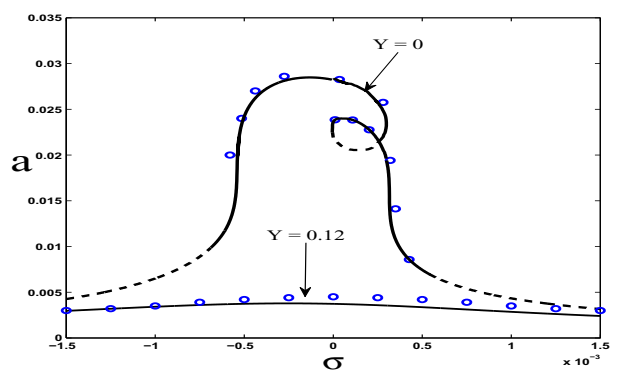

Fig. 6. Amplitude versus $\sigma$ when $V=0.11, u_{1}=0.033, u_{2}=$ 0.033 and $v=8$. Solid line: stable, dashed line: unstable, circles: numerical simulation.

\section{Conclusion}

In this work, we have investigated the effect of FHE and IPD on the amplitude and the onset of periodic galloping of a tower when submitted to steady and turbulent wind flow. A lumped mass sdof model was considered and attention was focused on the case where the turbulent wind activates either external excitation, parametric one or both. The method of DPM and the MSM are used to drive a slow dynamic near primary resonance.

In the case of steady wind, the combined effect of FHE and IPD retards substantially the galloping onset and decreases significantly its amplitude.

In the case of turbulent wind, the FHE and IPD also decreases the amplitude of the galloping and increases the galloping onset of the tower in all cases of turbulent wind.

\section{Appendix}

The expressions of the coefficients of Eq. (1) are $\omega=\pi \frac{\sqrt{3 E I}}{h \ell \sqrt{m}}, c_{a}=\frac{\rho A_{1} b h \ell \bar{U}_{c}}{2 \pi \sqrt{3 E I m}}, b_{1}=c_{a}, b_{2}=-\frac{4 \rho A_{2} b_{1} \ell}{3 \pi m}$ $b_{31}=-\frac{3 \pi \rho A_{3} b \ell \sqrt{3 E I}}{8 h \bar{U}_{c} \sqrt{m^{3}}}, b_{32}=-b_{31}, \eta_{1}=\frac{4 \rho A_{0} b h^{2} \ell \bar{U}_{c}^{2}}{3 \pi^{3} E I}$ $\eta_{2}=\frac{\eta_{1}}{2}, U(t)=\bar{U}+u(t)$

where $\ell$ is the height of the tower, $b$ is the cross-section wide, $E I$ the total stiffness of the single story, $m$ is the mass longitudinal density, $h$ is the inter story height, and $\rho$ is the air mass density.

$A_{i}, i=0, \ldots 3$ are the aerodynamic coefficients for the squared cross-section.

The dimensional critical velocity is given by

$\bar{U}_{c}=\frac{4 \pi \xi \sqrt{3 E I m}}{\rho b A_{1} h \ell}$.

Here $\xi$ is the modal damping ratio, depending on both the external and internal dampings:

$\xi=\frac{\eta h^{2}}{24 E I} \omega+\frac{c}{2 m \omega}$

\section{References}

1. G.V. Parkinson, J.D. Smith, The square prism as an aeroelastic non-linear oscillator. Quarterly Journal of Mechanics and Applied Mathematics 17, (1964) 225-239

2. M. Novak, Aeroelastic galloping of prismatic bodies. ASCE, Journal of Engineering Mechanics Division 96, (1969) 115-142

3. A.H. Nayfeh, M. Abdel-Rohman, Galloping of squared cantilever beams by the method of multiple scales. J. Sound Vib. 143, (1990) 87-93

4. M. Abdel-Rohman, Effect of unsteady wind flow on galloping of tall prismatic structures, Nonlinear Dyn. 26, (2001) 231-252

5. R. Clark, A. Modern, Course in Aeroelasticity, fourth ed (Kluwer Academic Publishers, Dordrecht, The Netherlands, 2004)

6. B.F. Spencer Jr, S. Nagarajaiah, State of the art of structural control. Journal of Structural Engineering, 129, (2003) 845-865

7. A. H. Nayfeh, B. Balachandran, Applied Nonlinear Dynamics (John Wiley, New York, 1995)

8. A. Luongo, D. Zulli, Parametric, external and selfexcitation of a tower under turbulent wind flow, J. Sound Vib. 330, (2011) 3057-3069

9. D. Zulli, A. Luongo, Bifurcation and stability of a twotower system under wind-induced parametric, external and self-excitation, J. Sound Vib. 331, (2012) 365-383

10. M.Belhaq, I. Kirrou, L. Mokni, Periodic and quasiperiodic galloping of a wind-excited tower under external excitation, Nonlinear Dyn. 74, (2013) 849-867

11. W.O. Keightley, G.W. Housner, D.E. Hudson, Vibration tests of the Encino dam intake tower, (California Institute of Technology, Report No. 2163, Pasadena, California 1961)

12. L. Mokni, I. Kirrou, M. Belhaq, Galloping of a windexcited tower under internal parametric damping, J. Vib. Acoust. (2014), doi: 10.1115/1.4026505

13. Munteanu, L., Chiroiu, V., Sireteanu, T.: On the response of small buildings to vibrations. Nonlinear Dyn. 73, (2013) 1527-1543

14. I. I. Blekhman, Vibrational Mechanics, Nonlinear Dynamic Effects, General Approach, Applications (Singapore: World Scientific 2000)

15. J.J. Thomsen, Vibrations and Stability: Advanced Theory, Analysis, and Tools (Springer- Verlag, BerlinHeidelberg 2003)

16. F. Lakrad, M. Belhaq, Suppression of pull-in instability in MEMS using a high-frequency actuation. Commun Nonlinear Sci Numer Simulat. 15, (2010) 3640-3646

17. I. Kirrou, L. Mokni, M. Belhaq, On the quasiperiodic galloping of a wind-excited tower, J. Sound Vib. 332 (2013) 4059-4066. 